\title{
Third Virial Coefficient for Air-Water Vapor Mixtures*
}

\author{
R. W. Hyland and E. A. Mason** \\ Institute for Basic Standards, National Bureau of Standards, Washington, D.C. 20234
}

(January 9, 1967)

\begin{abstract}
The third interaction virial coefficient $C_{a w w}$ for air-water vapor mixtures is estimated in the temperature range 0 to $100{ }^{\circ} \mathrm{C}$, by means of an approximate method based on molecular association. The results are believed accurate to within a factor of two. The value of $C_{\text {aww }}$ can be combined with previous estimates of the other interaction coefficient $C_{\text {aaw }}$ and the values of the third virial coefficients of pure air and pure water vapor, to give a quantitative description of the equation of state of air-water vapor mixtures through the complete third virial coefficient. It is shown that the maximum total contribution from both $C_{\text {aww }}$ and $C_{\text {aaw }}$ to the compressibility factor is only of the order of parts in $10^{4}$ up to $100{ }^{\circ} \mathrm{C}$ and 100 atmospheres total pressure. At $-50{ }^{\circ} \mathrm{C}$, it can be shown by extrapolation of $C_{a w w}$ that the contribution of the $C_{a w w}$ term to the compressibility factor is only on the order of parts in $10^{11}$.
\end{abstract}

Key Words: Chemical association, Lennard-Jones potential parameters, virial coefficients, water vapor-air mixtures.

\section{Introduction}

The thermodynamic properties of air-water vapor mixtures near atmospheric pressure have been determined and tabulated by Goff and his co-workers [1-4]. ${ }^{1}$ Close correlation was found between the equation of state in the virial form and experimental observations; since the virial form has a sound theoretical basis, Goff was able to make accurate interpolations and extrapolations. In this work, Goff neglected terms involving the third interaction virial coefficients $C_{\text {aaw }}$ and $C_{a w w}$, which account respectively for interactions among two air molecules and one water molecule, and one air molecule and two water molecules. This neglect was dictated at least in part by necessity, since no information on $C_{a a w}$ and $C_{a w w}$ was available at that time, but the good agreement between theory and experiment indicated that the neglect was probably justified. However, the effects of $C_{a a w}$ and $C_{a w w}$ increase with increasing pressure, and it cannot be taken for granted that they can be neglected at high pressures, where interactions among three molecules become important. The situation was improved somewhat in 1963, when Mason and Monchick [5] presented theoretical estimates of $C_{a a w}$, believed accurate within a factor of two. Only $C_{a w w}$ then remained unknown in the treatment of the equation of state of moist air through the third virial coefficient.

The purpose of this paper is to present theoretical estimates of $C_{a w w}$. Internal evidence in the calcula- tions indicates that the results are reliable within a factor of two from 0 to $100{ }^{\circ} \mathrm{C}$; even order of magnitude reliability would be valuable, however, because no other information whatever is available on $C_{a w w}$. The results can be extrapolated down to $-50{ }^{\circ} \mathrm{C}$ with no loss in overall accuracy in the equation of state, since the maximum water content of moist air is so small at low temperatures. The results can also be extrapolated above $100{ }^{\circ} \mathrm{C}$, but the upper limit of usefulness is uncertain. At $100{ }^{\circ} \mathrm{C}$ and pressures up to $100 \mathrm{~atm}$, however, the total contribution from both $C_{\text {aaw }}$ and $C_{a w w}$ to the compressibility factor is only of the order of parts in $10^{4}$, so considerable extrapolation is possible if only moderately accurate equation of state information is needed.

The rigorous calculation of $C_{a w w}$ is difficult because one of the pair interactions, $w w$, is qualitatively different from the other two, aw, inasmuch as a longrange dipole-dipole force is involved. However, if the dipole-dipole interaction is strong enough, a good approximation can be obtained by assuming that the two water molecules form a quasi-stable dimer, $w_{2}$, whose interaction with a single air molecule is relatively easy to calculate. This is the physical basis for the present theoretical estimates.

\section{The Virial Equation of State}

The virial equation of state through the third virial coefficient for a mixture is

$$
\frac{P V}{R T}=1+\frac{B_{\text {mix }}}{V}+\frac{C_{\text {mix }}}{V^{2}}+\ldots
$$

*This work was supported in part by the U.S. Army Research Office-Durham.

**Institute for Molecular Physics, University of Maryland, College Park, Md. 2074

'Figures in brackets indicate the literature references at the end of this paper. 
which can also be written as a series in the pressure,

$\frac{P V}{R T}=1+B_{\text {mix }} \frac{P}{R T}+\left(C_{\text {mix }}-B_{\text {mix }}^{2}\right)\left(\frac{P}{R T}\right)^{2}+\ldots$

where

$$
\begin{aligned}
P & =\text { absolute pressure, atm, } \\
V & =\text { molar volume of mixture, } \mathrm{cm}^{3} / \mathrm{mol}, \\
T & =\text { absolute temperature, }{ }^{\circ} \mathrm{K}, \\
R & =\text { gas constant, } \mathrm{cm}^{3} \text {-atm } / \mathrm{mol}^{\circ} \mathrm{K}, \\
B_{\text {mix }} & =\text { second virial coefficient of the mixture, } \\
& \mathrm{cm}^{3} / \mathrm{mol}, \text { and }
\end{aligned}
$$

The virial coefficients of a mixture depend on both temperature and composition, but the composition dependence is simple. For a binary mixture of $a$ and $w$, it is

$$
\begin{aligned}
& B_{\text {mix }}=x_{a}^{2} B_{a a}+2 x_{a} x_{w} B_{a w}+x_{w}^{2} B_{w w} \\
& C_{\text {mix }}=x_{a}^{3} C_{a a a}+3 x_{a}^{2} x_{w} C_{a a w}+3 x_{a} x_{w}^{2} C_{a w w}+x_{w}^{3} C_{w w w}
\end{aligned}
$$

where

$$
\begin{aligned}
x_{a}, x_{w}= & \text { mole fractions of air and water vapor } \\
& \text { respectively, } \\
B_{a a}, B_{w w}= & \text { second virial coefficients of pure air and } \\
& \text { pure water vapor, respectively, } \\
B_{a w}= & \text { second interaction virial coefficient for } \\
& \text { one air molecule and one water } \\
& \text { molecule, } \\
C_{a a a}, C_{w w w}= & \text { Third virial coefficients of pure air and } \\
& \text { pure water vapor, respectively, and } \\
C_{\text {aaw }}, C_{a w w}= & \text { third interaction virial coefficients for } \\
& \text { two air molecules and one water } \\
& \text { molecule and for one air molecule } \\
\text { and two water molecules, respec- } & \text { tively. }
\end{aligned}
$$

All the $B_{i j}$ and $C_{i j k}$ are functions of temperature only, and are independent of composition.

\section{Association Treatment of Virial Coefficients}

Our approximation is based on the treatment of the equation of state in terms of chemical association, as has been discussed, for example, by Woolley [6]. In this treatment, a single-component gas is considered to be an ideal mixture of molecular clusters consisting of monomers, dimers, trimers, etc. The actual nonideal behavior of the gas is thus accounted for by the change in the total number of molecules due to the association-an ideal gas would consist only of monomers. This treatment leads to the following equation of state for one mole of a single-component gas:

$$
\frac{P V}{R T}=1-K_{2} P+\left(3 K_{2}^{2}-2 K_{3}\right) P^{2}+\ldots
$$

where $K_{2}$ is the equilibrium constant for dimer formation, $K_{3}$ that for trimer formation, and so on.

At this point the results are completely rigorous, and in fact there is a one-to-one correspondence between the equilibrium constants and the Mayer cluster integrals of statistical-mechanical theory. Approximations enter only in the evaluation of the equilibrium constants, and the chemical association method is most useful in those cases where the interactions are strong enough that $K_{2}$, for instance, can be obtained by considering only those pairs of molecules which are bound together in their lowest energy state. If all states of binding are considered, then the ordinary virial equation of state is recovered, but couched in somewhat different language than usual.

For example, for pure water vapor, treated as a mixture of monomers, dimers, etc., comparison of eq (5) and the virial equation of state for water monomer shows that

$$
B_{w w}=-K_{2} R T, C_{w w w}=\left(4 K_{2}^{2}-2 K_{3}\right)(R T)^{2}, \text { etc. }
$$

According to the law of mass action,

$$
K_{2}=\frac{x_{w_{2}} V}{x_{w}^{2} R T}
$$

where $\mathbf{x}_{w_{2}}$ is the mole fraction of dimers and $x_{w}$ the mole fraction of monomers. Combination of eqs (6) and (7) leads to

$$
B_{w w}=-\frac{x_{w_{2}} V}{x_{w}^{2}} \text { or } \frac{B_{w w}}{V}=-\frac{x_{w_{2}}}{x_{w}^{2}}
$$

and $\quad \frac{P V}{R T}=1-\frac{x_{w_{2}}}{x_{w}^{2}}+\ldots \approx 1-x_{w_{2}}+\ldots$

for $x_{w_{2}} \ll x_{w}$.

The treatment is easily extended to gas mixtures, and a hybrid description is even possible, in which strongly interacting components of the mixture are handled by the chemical association method, and weakly interacting components by the ordinary virial coefficient method. Such a hybrid description is appropriate for mixtures of air and water vapor. We will not, however, calculate $B_{w w}$ from eq (6), but will take $B_{w w}$ as an experimental quantity and use eq (8) to calculate the mole fraction of dimers, which will be needed in the estimation of $C_{\text {aww }}$.

Barua, et al. [7] have shown that for polar molecules a fairly good approximation to $B$ and $K_{2}$ can be obtained by considering only those pairs of molecules which interact strongly enough to be bound together 
in the lowest energy state-that is, by considering only dimers whose component molecules have their dipoles alined head-to-tail. The contributions from other dimer configurations can be ignored if the temperature is not too high. This study thus gives quantitative evidence for the validity of the concept of dimers of water molecules.

For a description only through the third virial coefficient, we can consider the three-molecule interactions either as trimer formation, or else as the nonideal interaction between a dimer and a monomer. Our model of a mixture of air and water vapor through the third virial coefficient will be that of a mixture of three species-air monomers, water monomers, and water dimers. All of these species interact with one another except for two water monomers, whose interaction is already taken into account by the existence of the water dimers. The equation of state of this ternary mixture is

$$
\begin{aligned}
\frac{P V}{R T}=1+ & \left(x_{a}^{\prime 2} B_{a a}+2 x_{a}^{\prime} x_{w}^{\prime} B_{a w}+x_{w}^{\prime 2} B_{w w}\right) \frac{1}{V} \\
& +\left(2 x_{a}^{\prime} x_{w_{2}} B_{a w_{2}}+2 x_{w}^{\prime} x_{w_{2}} B_{w w_{2}}\right) \frac{1}{V} \\
& +\left(x_{a}^{\prime 3} C_{a a a}+3 x_{a}^{\prime 2} x_{w}^{\prime} C_{a a w}\right)\left(\frac{1}{V}\right)^{2}+\ldots
\end{aligned}
$$

in which we have written $B_{w w}$ explicitly rather than use eq (8), since we will take $B_{w w}$ as an experimental quantity. Other interactions, such as those involving $B_{w_{2} w_{2}}$ or $C_{a a w_{2}}$, contribute to the fourth virial coefficient and are neglected. The mole fractions have been written with primes to indicate that they are not the same as those appearing in eqs (3) and (4).

The degree of dimerization is on the order of parts per thousand of the total number of water molecules. We therefore make the approximation that the $x_{a}^{\prime}$ and $x_{w}^{\prime}$ of eq (10) are equal to the $x_{a}$ and $x_{w}$ of eqs (3) and (4). By comparison of these equations, we see that

$$
\begin{aligned}
C_{\text {mix }}=\left(2 x_{a} x_{w_{2}} B_{a w_{2}}+2 x_{w} x_{w_{2}} B_{w w_{2}}\right) V & \\
& +\left(x_{a}^{3} C_{a a a}+3 x_{a}^{2} x_{w} C_{a a w}\right) .
\end{aligned}
$$

Solving eq (8) for $x_{w_{2}}$ and substituting into the first term on the right, we find

$$
3 x_{a} x_{w}^{2} C_{a w w}+x_{w}^{3} C_{w w w}=-2 x_{a} x_{w}^{2} B_{a w_{2}} B_{w w}-2 x_{w}^{3} B_{w w_{2}} B_{w w}
$$

and therefore

$$
\begin{gathered}
C_{a w w}=-\frac{2}{3} B_{a w_{2}} B_{w w} \\
C_{w w w}=-2 B_{w w_{2}} B_{w w} .
\end{gathered}
$$

The quantity sought, $C_{a w w}$, has therefore been related to a product of second virial coefficients, one of which $\left(B_{w w}\right)$ is known experimentally, and the other of which $\left(\boldsymbol{B}_{a w_{2}}\right)$ remains to be calculated. In addition, if $C_{w w w}$ is known experimentally, we can use eq (14) as a check on the internal consistency of our above calculations or as a source of information on the dimer properties (through $B_{w w_{2}}$ ).

\section{Calculation of $B_{w w}$}

We can now calculate values of $B_{w w}$, from eq (14), and compare with values obtained by methods described in this section. This affords a check on our procedures to this point.

It must be emphasized that we are not trying to derive values for $B_{w w_{2}}$ as an end in itself-rather, we wish to determine the ranges within which the molecular potential parameters for the dimer-monomer interaction lie. This is important, for these parameters will be used to obtain, in order, the molecular potential parameters associated with the air-dimer interaction, $B_{a w_{2}}$ itself, and finally $C_{a w w}$ from eq (13).

The values of $B_{w w}$ and $C_{w w w}$ are listed in table 1 , as taken from the correlations of Goff [4] and Keyes [8]. The respective values of $B_{w w}$ warrant using the average value in all calculations. The respective $C_{w w w}$ values, however, should be considered individually. Goff [4] assigns a tolerance of \pm 120 percent at $60{ }^{\circ} \mathrm{C}$ and of

\begin{tabular}{|c|c|c|c|c|c|}
\hline \multirow{2}{*}{ Temp. } & \multicolumn{3}{|c|}{$\begin{array}{c}-B_{w w} \\
\mathrm{~cm}^{3} / \mathrm{mol}\end{array}$} & \multicolumn{2}{|c|}{$\begin{array}{c}-C_{\text {wux }} \\
\text { liter }^{2} / \mathrm{mol}^{2}\end{array}$} \\
\hline & Goff [4] & Keyes [8] & Ave. & Goff [4] & Keyes [8] \\
\hline${ }^{\circ} \mathrm{C}$ & & & & & \\
\hline 0 & (1832) & 1854 & 1843 & $(64.3)$ & 93.8 \\
\hline 10 & 1510 & 1520 & 1515 & $(34.7)$ & 49.3 \\
\hline 20 & 1260 & 1266 & 1263 & (19.7) & 27.6 \\
\hline 30 & 1074 & 1070 & 1072 & (11.7) & 16.2 \\
\hline 40 & 924 & 917 & 920 & (7.18) & 9.87 \\
\hline 50 & 803 & 794 & 798 & (4.51) & 6.26 \\
\hline 60 & 705 & 695 & 700 & 3.01 & 4.06 \\
\hline 70 & 625 & 613 & 619 & 2.03 & 2.76 \\
\hline 80 & 558 & 546 & 552 & 1.39 & 1.92 \\
\hline 90 & 501 & 489 & 495 & 0.98 & 1.37 \\
\hline 100 & (453) & 441 & 447 & $(.70)$ & 0.99 \\
\hline
\end{tabular}
\pm 30 percent at $90{ }^{\circ} \mathrm{C}$ to his values of $C_{w w w}$, with the tolerances taken as twice the probable error. This points out the large uncertainty in this quantity, particularly when we have used Goff's relations to extrapolate his values below $60{ }^{\circ} \mathrm{C}$.

TABLE 1. Experimental values of the second and third virial coefficients of water vapor; extrapolated values in parentheses

Two sets of $B_{w w,}$ values are calculated from eq (14), one set from Goff's $C_{w w w}$, the other from Keyes' $C_{w w w}$. The results are given in table 2 .

Other estimates may be obtained from assumptions about the intermolecular forces. We represent the interaction between two molecules as the sum of a spherically symmetric Lennard-Jones (12-6) interaction and the interaction between point dipoles embedded in the center of each molecule. The potential 
energy $\varphi_{i j}(r)$ between molecules $i$ and $j$ a distance $r$ apart is thus

$$
\begin{aligned}
\varphi_{i j}(r) & =4 \epsilon_{i j}\left[\left(\frac{\sigma_{i j}}{r}\right)^{12}-\left(\frac{\sigma_{i j}}{r}\right)^{6}\right] \\
& -\frac{\mu_{i} \mu_{j}}{r^{3}}\left(2 \cos \theta_{i} \cos \theta_{j}-\sin \theta_{i} \sin \theta_{j} \cos \phi\right)
\end{aligned}
$$

where $\mu_{i}$ and $\mu_{j}$ are the dipole moments, $\theta_{i}$ and $\theta_{j}$ are the angles between the dipole axes and the line of centers of the molecules, $\phi$ is the azimuth angle of the axes, and $\epsilon_{i j}$ and $\sigma_{i j}$ are adjustable constants. If either dipole moment is zero, eq (15) reduces to the LennardJones (12-6) potential, for which $\epsilon_{i j}$ is the depth of the potential well and $\varphi\left(\sigma_{i j}\right)=0$. Equation (15) is usually called the Stockmayer potential [9].

TABLE 2. Experimental and calculated values of the water monomer-

\begin{tabular}{|c|c|c|c|c|c|c|}
\hline \multirow{2}{*}{ Temp. } & \multicolumn{6}{|c|}{$-B_{u x u_{2}}$ liter $/ \mathrm{mol}$} \\
\hline & \multicolumn{2}{|c|}{$\begin{array}{c}\text { Exptl., eq (14) } \\
\left.\text { (Keyes' } C_{u w u}\right)\left(G_{\left.\text {Goff's } C_{u w u}\right)}\right.\end{array}$} & \multirow[t]{2}{*}{$\begin{array}{l}\text { Calc. } \\
\text { eq (18) }\end{array}$} & \multirow[t]{2}{*}{$\begin{array}{l}\text { Calc. } \\
\text { eq (19) }\end{array}$} & \multirow[t]{2}{*}{$\begin{array}{l}\text { Calc. } \\
\text { eq (2la) }\end{array}$} & \multirow[t]{2}{*}{$\begin{array}{l}\text { Calc. } \\
\text { eq }(21 \mathrm{~b})\end{array}$} \\
\hline${ }^{\circ} \mathrm{C}$ & & & & & & \\
\hline 0 & 25.4 & 17.4 & 21.53 & 11.91 & 3.39 & 5.08 \\
\hline 10 & 16.3 & 11.5 & 17.52 & 9.84 & 2.66 & 3.99 \\
\hline 20 & 11.0 & 7.80 & 12.96 & 8.20 & 2.25 & 3.15 \\
\hline 30 & 7.56 & 5.46 & 10.44 & 6.72 & 1.89 & 2.56 \\
\hline 40 & 5.36 & 3.90 & 8.21 & 6.13 & 1.62 & 2.20 \\
\hline 50 & 3.92 & $2.8^{\circ}$ & 6.61 & 4.84 & 1.37 & 1.82 \\
\hline 60 & 2.90 & 2.1 i & 5.58 & 4.22 & 1.21 & 1.55 \\
\hline 70 & 2.23 & 1.64 & 4.27 & 3.67 & 1.09 & 1.36 \\
\hline 80 & 1.74 & 1.26 & 3.65 & 3.16 & 0.96 & 1.18 \\
\hline 90 & 1.38 & 0.99 & 3.10 & 2.73 & .86 & 1.06 \\
\hline 100 & 1.11 & .79 & 2.63 & 2.46 & .79 & 0.95 \\
\hline
\end{tabular}
dimer second virial coefficient

The values of $\epsilon_{i j}$ and $\sigma_{i j}$ must be determined from some experimentally measured property. For pure air, which has no dipole moment, the values used by Mason and Monchick [5] and by Chaddock [10] are $\sigma_{a a}=3.522 \AA$ and $\epsilon_{a a} / k=99.2{ }^{\circ} \mathrm{K}$, where $k$ is Boltzmann's constant. For pure water vapor, Rowlinson [11] gives $\sigma_{w w}=2.65 \AA$ and $\epsilon_{w w} / k=380{ }^{\circ} \mathrm{K}$ from analysis of $B_{w w}$, and Monchick and Mason [12] give $\sigma_{w w}=2.71 \AA$ and $\epsilon_{w w} / k=506{ }^{\circ} \mathrm{K}$ from analysis of viscosity data. Tabulations of virial coefficients for the Stockmayer potential are given in terms of a reduced dipole parameter, defined as

$$
t_{i j}^{*}=\frac{\mu_{i} \mu_{j}}{\sqrt{8} \epsilon_{i j} \sigma_{i j}^{3}} .
$$

The value of $t_{w w}^{*}$ is 1.2 according to Rowlinson and 0.85 according to Monchick and Mason, with the dipole moment of water taken as $\mu_{w}=1.83 \times 10^{-18}$ esu.

Stogryn and Hirschfelder [13] have shown how to estimate $\epsilon_{i j}$ and $\sigma_{i j}$ for dimer-monomer interactions from those for monomer-monomer interactions, according to simple geometrical arguments. The results have been elaborated by Barua and Das Gupta [13] on the basis of the pressure dependence of the viscosity of superheated steam. They recommend the values

$$
\epsilon_{w w_{2}}=1.32 \epsilon_{w w}, \sigma_{w w_{2}}=1.16 \sigma_{w w} \text {. }
$$

Since we assume that the dimer consists of two monomers with their dipoles in a head-to-tail configuration, the dipole moment of the dimer is twice that of the monomer, $\mu_{w_{2}}=3.66 \times 10^{-18}$ esu.

Once $\epsilon, \sigma$, and $t^{*}$ are known, it is straightforward to calculate $B_{w w_{2}}$ from published numerical tables $[9,11]$ for the Stockmayer potential.

The Rowlinson [11] parameters for water vapor, based on virial coefficient data, are considered first, since so far only virial coefficient data have been used in this presentation.

Equations (16) and (17) lead to

$$
t_{w w_{2}}^{*}=1.2, \sigma_{w w_{2}}=3.07 \AA, \epsilon_{w w_{2}} / k=501^{\circ} \mathrm{K} .
$$

The values of $\boldsymbol{B}_{w w_{2}}$ obtained from these parameters are found in table 2. The agreement between the values of $-B_{a w_{2}}$ calculated from eqs (14) and (18) is generally good, although the latter are somewhat higher except at $0{ }^{\circ} \mathrm{C}$. The implication is that the assumptions made to derive our model are at least as reasonable an approximation of the true physical situation as those used in the derivation of the Stogryn and Hirschfelder [13] model.

If the viscosity-derived parameters of Mason and Monchick [5] for water vapor are used with eqs (16) and (17), we find

$t w w_{2}=0.83, \sigma_{w w_{2}}=3.14 \AA, \epsilon_{w w_{2}} / k=668{ }^{\circ} \mathrm{K}$.

The values of $B_{w w_{2}}$ derived from these parameters are also given in table 2. The curve of this set of $B_{w w_{2}}$ values versus $T$ is flatter at the low temperature end than the three curves obtained above from virial coefficient data, but the agreement still is not unreasonable.

Since the values of $B_{w w_{2}}$ derived from eq (14) do agree fairly well with those derived from other sources, we now seek, by curve fitting, improved Stockmayer potential parameters which will describe the values from eq (14). These will be needed in the calculation of $C_{a w w}$.

The curve-fitting should be done so as to produce preferentially better agreement at high temperatures than at low temperatures for two reasons. First, the experimental values of $C_{w w w}$ are less uncertain at high temperatures. Second, and more important, is the fact that the low-temperature values of $B_{w w_{2}}$ are dominated more by $t_{w w_{2}}^{*}$ than are the high-temperature values, but the parameter $t_{w w_{2}}^{*}$ is of no interest for the present purposes, since it does not contribute at all to the determination of $C_{a w w}$. In other words, we want the derived values of $\epsilon_{w w_{2}}$ and $\sigma_{w w_{2}}$ to represent the spherical (12-6) part of the potential, not the dipole part, as there is no dipole contribution to $B_{a w_{2}}$.

Therefore, we require that the $\boldsymbol{B}_{w w_{2}}$ values obtained by fitting should lie between $-0.79 \mathrm{liter} / \mathrm{mol}$ and -1.11 liter/mole at $100{ }^{\circ} \mathrm{C}$, that is, between the values predicted respectively by using Goff's and Keyes' $C_{w w w}$ in eq (14). At the same time the slopes of the curves in the $100{ }^{\circ} \mathrm{C}$ region must be almost the same to prevent any gross divergences at higher temperatures. 
The limiting ranges of $\sigma_{w w_{2}}$ and $\epsilon_{w w_{2}}$ are set arbitrarily, but not unreasonably, in the following manner. The lower limits are taken to be the corresponding values for pure water vapor given by Rowlinson [11]. This is equivalent to setting the coefficients of $\sigma_{w w}$ and $\epsilon_{w v}$ in eq (17) equal to 1 , a change of 13.8 percent in the former and 24.2 percent in the latter. The upper limits are set by raising the coefficients by the same percentages, and using the $\sigma_{w w}$ and $\epsilon_{w w}$ parameters of Mason and Monchick [5] in eq (17). The limits on $t_{w w_{2}}^{*}$ are found by using the limiting values of $\epsilon_{w w_{2}}$ and $\sigma_{w w_{2}}$ in eq (16).

This procedure leads to

$$
\begin{aligned}
& \sigma_{w w_{2}} \geqslant\left(\sigma_{w w}\right)_{\text {Rowlinson }}=2.65 \AA, \\
& \epsilon_{w w_{2}} / k \geqslant\left(\epsilon_{w w} / k\right)_{\text {Rowlinson }}=380^{\circ} \mathrm{K}, \\
& t_{w w_{2}}^{*} \leqslant 2.4 \\
& \sigma_{w w_{2}} \leqslant 1.32\left(\sigma_{w w}\right)_{\text {M. and м. }}=3.58 \AA, \\
& \epsilon_{w w_{2}} / k \leqslant 1.64\left(\epsilon_{w w} / k\right)_{\text {M. and м. }}=830^{\circ} \mathrm{K}, \\
& t_{w w_{2}}^{*} \geqslant 0.45 .
\end{aligned}
$$

Within these ranges, the predicted curves are slightly flatter in the $100{ }^{\circ} \mathrm{C}$ region than those predicted by eq (14). This means that a curve which predicts - 1.11 liter/mole at $100{ }^{\circ} \mathrm{C}$ will diverge immediately from higher temperature $B_{w w_{2}}$ values derived from Keyes' $C_{w w w}$. In order to prevent this immediate divergence, we changed the lower permissible $B_{w w}$. limit at $100{ }^{\circ} \mathrm{C}$ Irom -1.11 liter $/ \mathrm{n}$.u1 to -0.95 liter $/ \mathrm{mol}$, that is, miaway between the limits set by eq (14).

Empirical curve fitting with $B_{w w_{2}}$ matching at both $-0.79 \mathrm{liter} / \mathrm{mol}$ and $-0.95 \mathrm{liter} / \mathrm{mol}$ at $100{ }^{\circ} \mathrm{C}$ led respectively to these choices of parameters:

$t_{w w_{2}}^{*}=1.14, \sigma_{w w_{2}}=3.41 \AA, \epsilon_{w w_{2}} / k=380{ }^{\circ} \mathrm{K}$

$t_{w w_{2}}^{*}=1.25, \sigma_{w w_{2}}=3.30 \AA, \epsilon_{w w_{2}} / k=380{ }^{\circ} \mathrm{K}$.

The $\sigma_{w w_{2}}$ values are not unreasonable. The fact that $\epsilon_{w w_{2}} / k$ is the same as the value for pure water vapor (lower limit) in both cases is perhaps annoying, but can be accepted in the present context without undue alarm.

The values of $B^{*}$ versus $T^{*}$ for the parameters were interpolated graphically between tabulated values of $t^{*}[9]$, and these in curn were extrapolated graphically to the lowest temperatures. Therefore the derived parameters may not be the "best" ones in a least squares sense, but they are certainly close enough for our purposes.

The values of $B_{w w_{2}}$ corresponding to eqs (2la) and (2lb) are given in table 2 , and clearly the agreement with the curves derived from eq (14) is poor except at the highest temperatures. The reason for the discrep- ancy is open to some speculation, but we remind the reader again that first, we demanded a fit at the high temperatures to have the $\sigma$ and $\epsilon$ values be more representative of the spherical part of the potential, and second, we are seeking various estimates of $\sigma_{w w_{2}}$ and $\epsilon_{w w_{2}}$ rather than attempting to derive $B_{w w_{2}}$.

Summarizing this section, we have obtained four sets of $\sigma_{w w_{2}}$ and $\epsilon_{w w_{2}}$ parameters ( $t^{*}$ is of no further interest). Two were obtained through the method of Stogryn and Hirschfelder [13], using first experimental virial coefficient data and then experimental viscosity data, and two from curve-fitting, subject to several restraints, to the $\boldsymbol{B}_{w w_{2}}$ values derived with the methods of this paper. We also obtained what are thought to be reasonable outside limits on the values of $\sigma_{w w_{2}}$ and $\epsilon_{w w_{2}}$.

\section{Calculation of $C_{\text {aww }}$}

The various values of $\sigma_{w w_{2}}$ and $\epsilon_{w w_{2}}$ derived in the previous section may now be used to obtain the parameters $\sigma_{a w_{2}}$ and $\epsilon_{a w_{2}}$ for the air-dimer interaction, from which $B_{a w_{2}}$ and finally $C_{a w w}$ may be obtained. The procedure is based on semi-empirical combining rules [9] for $\epsilon$ and $\sigma$,

$$
\epsilon_{i j}=\left(\epsilon_{i i} \epsilon_{j j}\right)^{1 / 2}, \sigma_{i j}=(1 / 2)\left(\sigma_{i i}+\sigma_{j j}\right) .
$$

These rules work fairly well for both polar and nonpolar molecules [15]. Applying these rules twice, we obtain expressions for the desired parameters $\epsilon_{a w_{2}}$ and $\sigma_{a w_{2}}$

$$
\begin{aligned}
& \epsilon_{a w_{2}}=\epsilon_{w w_{2}}\left(\epsilon_{a a}^{\dot{a}} / \epsilon_{w w}\right)^{1 / 2}, \\
& \sigma_{a w_{2}}=\sigma_{w w_{2}}+(1 / 2)\left(\sigma_{a a}-\sigma_{w w}\right) .
\end{aligned}
$$

The values of $\sigma_{a w_{2}}$ and $\epsilon_{a w_{2}}$ are derived from the four sets of $w w_{2}$ parameters obtained in the previous section. For the three associated with virial coefficient data [eqs (18), (2la), (21b)] we use in eq (23) Rowlinson's [11] values for $\epsilon_{w w}$ and $\sigma_{w w}$, while for the set associated with viscosity data [eq (19)] we use in eq (23) the Mason and Monchick [5] values. We find

$$
\begin{array}{ll}
\epsilon_{a w_{2}} / k=256^{\circ} \mathrm{K} & \sigma_{a w_{2}}=3.51 \AA[\text { from eq (18)] } \\
\epsilon_{a w_{2}} / k=194{ }^{\circ} \mathrm{K} & \sigma_{a w_{2}}=3.85 \AA[\text { from eq (2la)] } \\
\epsilon_{a w_{2}} / k=194{ }^{\circ} \mathrm{K} & \sigma_{a w_{2}}=3.74 \AA[\text { from eq }(21 \mathrm{~b})] \\
\epsilon_{a w_{2}} / k=296^{\circ} \mathrm{K} & \sigma_{a w_{2}}=3.55 \AA[\text { from eq (19)] }
\end{array}
$$

The last set is of particular interest, since it is derived entirely from viscosity data. The viscosity is relatively insensitive to the dipole interaction [12], so the values of $\epsilon_{w w_{2}}$ and $\sigma_{w w_{2}}$ from which eq (24d) is derived should be good representations of the spherical part of the potential. 
Two more sets of $a w_{2}$ parameters should be derivednamely, those associated with the limiting cases of the $w w_{2}$ parameters [eqs (20a) and (20b)]. From these we obtain respectively

$$
\begin{aligned}
\boldsymbol{\epsilon}_{a w_{2}} / k & =194{ }^{\circ} \mathrm{K} & \sigma_{a w_{2}} & =3.09 \AA \\
\epsilon_{a w_{2}} / k & =423^{\circ} \mathrm{K} & \sigma_{a w_{2}} & =4.02 \AA .
\end{aligned}
$$

From each of these six sets of parameters, we calculate first $B_{a w_{2}}$ and then, using eq (13), $C_{a w w}$. The results of the latter are given in table 3 .

TABLE 3.-Calculated value of the third interaction virial coefficient $\mathrm{C}_{\mathrm{aww}}$ based on the parameters of eqs (24) and (25)

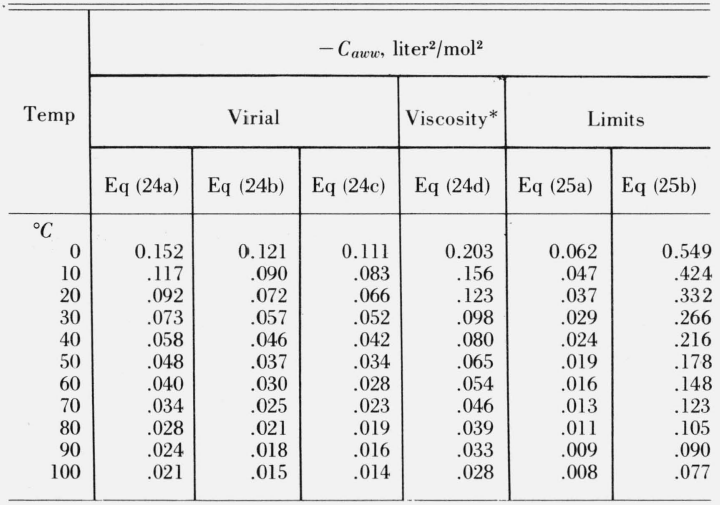

Those derived from experimental viscosity values [i.e., from eq (24)] are felt to be the most reliable. This is based on the relative independence of viscosity on the dipole interaction, and on the success of the dimermonomer model in predicting the pressure dependence of the viscosity of steam $[13,14]$.

The viscosity-based values differ from the limiting values derived for eq $(25 \mathrm{a}, \mathrm{b})$ by at most a factor of three, and from all other values by no more than a factor of two.

We are confident that the values are accurate to within a factor of four, and probably to within a factor of two.

\section{Discussion}

We are now in a position to estimate the contributions of $C_{\text {aaw }}$ and $C_{\text {aww }}$ to the compressibility factor of moist air; that is, to estimate the magnitudes of the terms $\left(3 x_{a}^{2} x_{w} C_{a a w} / V^{2}\right)$ and $\left(3 x_{a} x_{w}^{2} C_{a w w} / V^{2}\right)$, as compared to unity. Since the maximum water vapor content of air increases rapidly with increasing temperature, the largest contribution of these terms comes at high temperatures, even though $C_{a a w}$ and $C_{a w w}$ decrease in magnitude with increasing temperature. At $100{ }^{\circ} \mathrm{C}$ the partial pressure of water vapor can be taken as $1 \mathrm{~atm}$, and the mole fractions as

$$
x_{a}=P_{a} /\left(P_{a}+1\right), x_{w}=1 /\left(P_{a}+1\right)
$$

where $P_{a}$ is the partial pressure of the air. These relations are only approximate for nonideal gases, but adequate here because in $C_{\text {mix }}$ we are seeking only the highest-order deviation terms. Similarly, the molar volume of the mixture is

$$
V=R T /\left(P_{a}+1\right) .
$$

Taking $C_{\text {aaw }}=+696\left(\mathrm{~cm}^{3} / \mathrm{mol}\right)^{2}$ [5] and $C_{a w w}=-2.8$ $\times 10^{4}\left(\mathrm{~cm}^{3} / \mathrm{mol}\right)^{2}$ (table 3$)$, we find from eqs (26) and (27) that

$$
\begin{aligned}
& 3 x_{a}^{2} x_{w} C_{a a w} / V^{2}=2.23 \times 10^{-6} P_{a}^{2} /\left(P_{a}+1\right) \\
& 3 x_{a} x_{w}^{2} C_{a w w} / V^{2}=0.90 \times 10^{-4} P_{a} /\left(P_{a}+1\right) .
\end{aligned}
$$

Thus even for $P_{a}=100$ atm the contribution of these two terms is only of the order of parts in $10^{4}$.

The present results are readily extendable to higher or lower temperatures by means of the $a w_{2}$ parameters of eq (24d) in conjunction with tabulated virial coefficients for the (12-6) potential [9]. There is no loss in overall accuracy in $P V / R T$ by extrapolation to low temperatures. For example, a calculation similar to that performed above, based on a temperature of $-50{ }^{\circ} \mathrm{C}\left(P_{w}=3.9 \times 10^{-5}\right.$ atm $)$ with $P_{a}=100$ atm and $C_{\text {aww }}=-1 \times 10^{6}\left(\mathrm{~cm}^{3} / \mathrm{mol}\right)^{2}$, indicates that the contribution of the $C_{\text {aww }}$ term to the compressibility factor is on the order of parts in $10^{11}$. There is loss in accuracy by extrapolation to high temperatures because of the increasing water vapor content; how much extrapolation is permissible depends on how much uncertainty in $P V \mid R T$ is acceptable. It must also be remembered that the physical model of dimer formation becomes less accurate as the temperature increases.

\section{References}

[1] J. A. Goff and A. C. Bates, Trans. ASHVE 47, 373 (1941).

[2] J. A. Goff, J. R. Andersen, and S. Gratch, Trans. ASHVE 49, 269 (1943).

[3] J. A. Goff and S. Gratch, Trans. ASHVE 51, 125 (1945).

[4] J. A. Goff, Heating, Piping, and Air Conditioning (Journal Section) 21, 118 (1949).

[5] E. A. Mason and L. Monchick, Humidity and Moisture, Vol. III, p. 257 (Rheinhold Publishing Corp., New York, 1965).

[6] H. W. Woolley, J. Chem. Phys. 21, 236 (1953).

[7] A. K. Barua, P. K. Chakraborti, and A. Saran, Mol. Phys. 9, 9 (1965)

[8] F. G. Keyes, J. Chem. Phys. 15, 602 (1947).

[9] J. O. Hirschfelder, C. F. Curtiss, and R. B. Bird, Molecular Theory of Gases and Liquids (John Wiley \& Sons, Inc., New York, N.Y., second printing, 1964).

[10] J. B. Chaddock, Humidity and Moisture, Vol. III, p. 273 (Reinhold Publishing Corp., New York, 1965).

[11] J. S. Rowlinson, Trans. Faraday Soc. 45, 974 (1949).

[12] L. Monchick and E. A. Mason, J. Chem. Phys. 35, 1676 (1961).

[13] D. E. Stogryn and J. O. Hirschfelder, J. Chem. Phys. 31, 1531, 1545 (1959).

[14] A. K. Barua and A. Das Gupta, Trans. Faraday Soc. 59, 2243 (1963).

[15] E. A. Mason and L. Monchick, J. Chem. Phys. 36, 2746 (1962).

(Paper 71A3-454) 